\title{
Six challenges to stamping out Ebola
}

\section{Despite a recent sharp drop in the overall number of Ebola cases, the situation remains precarious in West Africa.}

\section{Declan Butler}

24 February 2015 I Corrected: 24 February 2015, 26 February 2015

More than a year since the start of one of the worst public health crises in recent history, Ebola cases have been tumbling in West Africa. But the epidemic is far from over: the risk of flare ups and further geographical spread will remain until there are no new cases.

The ease in case numbers means that public-health countermeasures and resources can be shifted in many places, from curbing runaway outbreaks to aggressively targeting the remaining, often smaller outbreaks. The region is also now vastly better prepared to tackle Ebola than it was five months ago, with greater levels of outbreak-response funding, infrastructure, staffing and experience. At the same time, there is a danger of complacency. Reducing the number of cases to zero demands identifying and breaking all new chains of transmission, a task that still faces major obstacles — not least the fast approaching rainy season.

Highlighting the precariousness of the current situation, on 20 February, the officials leading the United Nations' Ebola response efforts warned that the gains of the past few months risked unravelling.

Nature outlines six of the biggest challenges to stamping out Ebola.

\section{Precarious progress}

The sharp drop in the overall number of Ebola cases is a testament to the control efforts by the governments of Guinea, Liberia and Sierra Leone; the belated arrival of massive international assistance last autumn; and local populations who changed behaviours and practices to better control infection and spread (see 'Cases by country'). But the situation looks more precarious when you break it down by country and district.

The picture is brightest in Liberia, which has had fewer than five cases per week since 25 January. All cases in the past month have been confined to the county of Montserrado, which includes the capital, Monrovia, meaning that most of the country is now Ebola-free.

In Sierra Leone, however, the rapid drop in cases has faltered since the week ending 25 January, with a steady 60 to 80 cases every week since. Most of the new cases are in the capital Freetown, but worryingly, small numbers of cases also continue to occur in many districts throughout the country, meaning that the virus is still spread over a wide area and that many control interventions are needed.

Guinea also remains problematic. Since the epidemic began there in December 2013, community resistance to control measures such as tracing contacts of known or suspected cases and safe burials has badly hampered progress, leading to repeated resurgences. That pattern seems to be continuing, with cases having fallen to just 19 in the week ending 18 January, but then rebounding to just over 50 cases per week since the start of February. The most recent cases have been in the west of the country, in particular in the district of Forécariah, and the capital Conakry. 


\section{CASES BY COUNTRY}

The recent rapid fall in cases has stalled in Sierra Leone and Guinea and the situation is highly precarious.

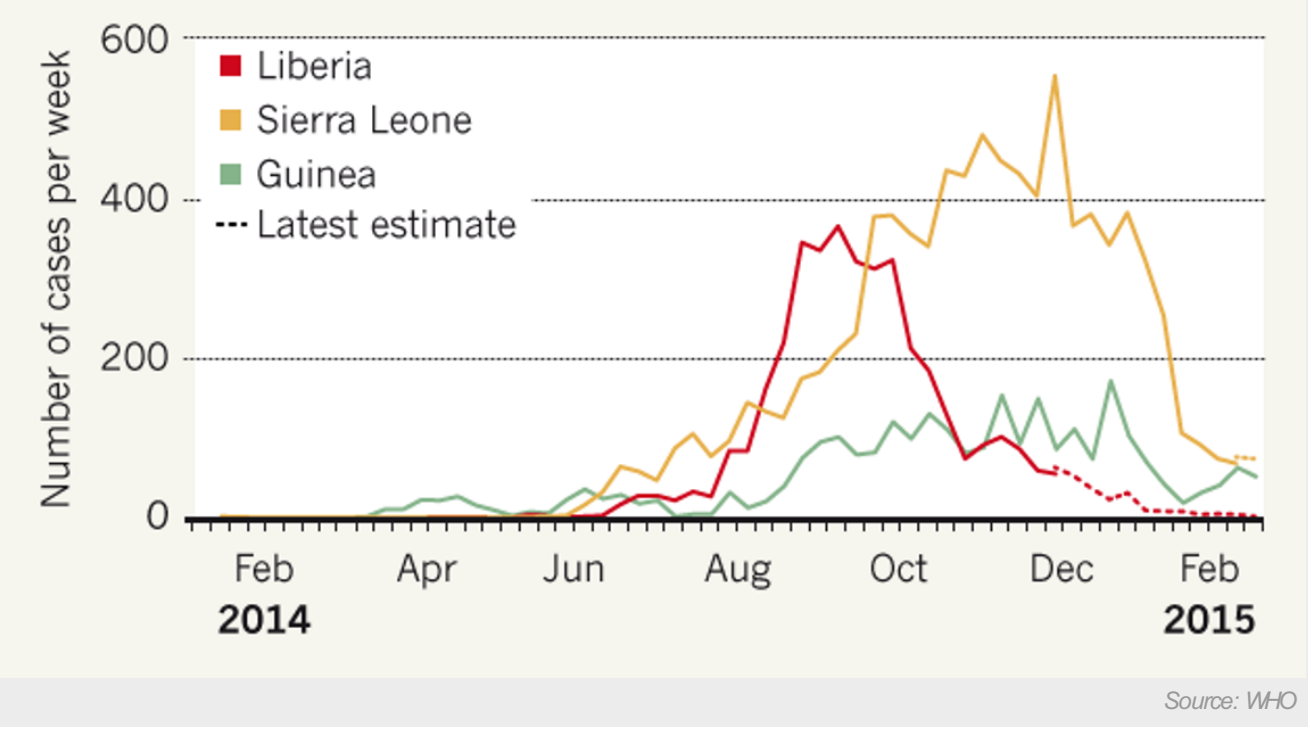

\section{Undetected transmission}

A major technique used by epidemiologists to slow spread and curb Ebola outbreaks is contact tracing. When someone is suspected of being infected, health workers isolate them, but also try to trace everyone that the person has recently had contact with and monitor these contacts for 21 days, the maxiumum incubation period of the disease. Contacts who develop Ebola are then in turn isolated and their contacts traced.

When the Ebola epidemic was in full swing, the sheer number of cases made contact tracing almost impossible. Now that numbers are much lower, the practice can, in principle, be pursued aggressively. But in Guinea and Sierra Leone, many new cases are still being reported in people who were not on existing contact lists (see 'Hidden disease spread'). That means that either not all contacts were identified, or that new transmission chains are going undetected. Exhaustive contact tracing requires large numbers of staff, and is challenging both in dense urban areas and in remote regions that are difficult to reach. Community resistance and the high mobility of populations pose further hurdles. 


\section{HIDDEN DISEASE SPREAD}

The high proportion of new Ebola cases in people who are not on the 'contact lists' of previously infected patients means that cases are still going undetected.

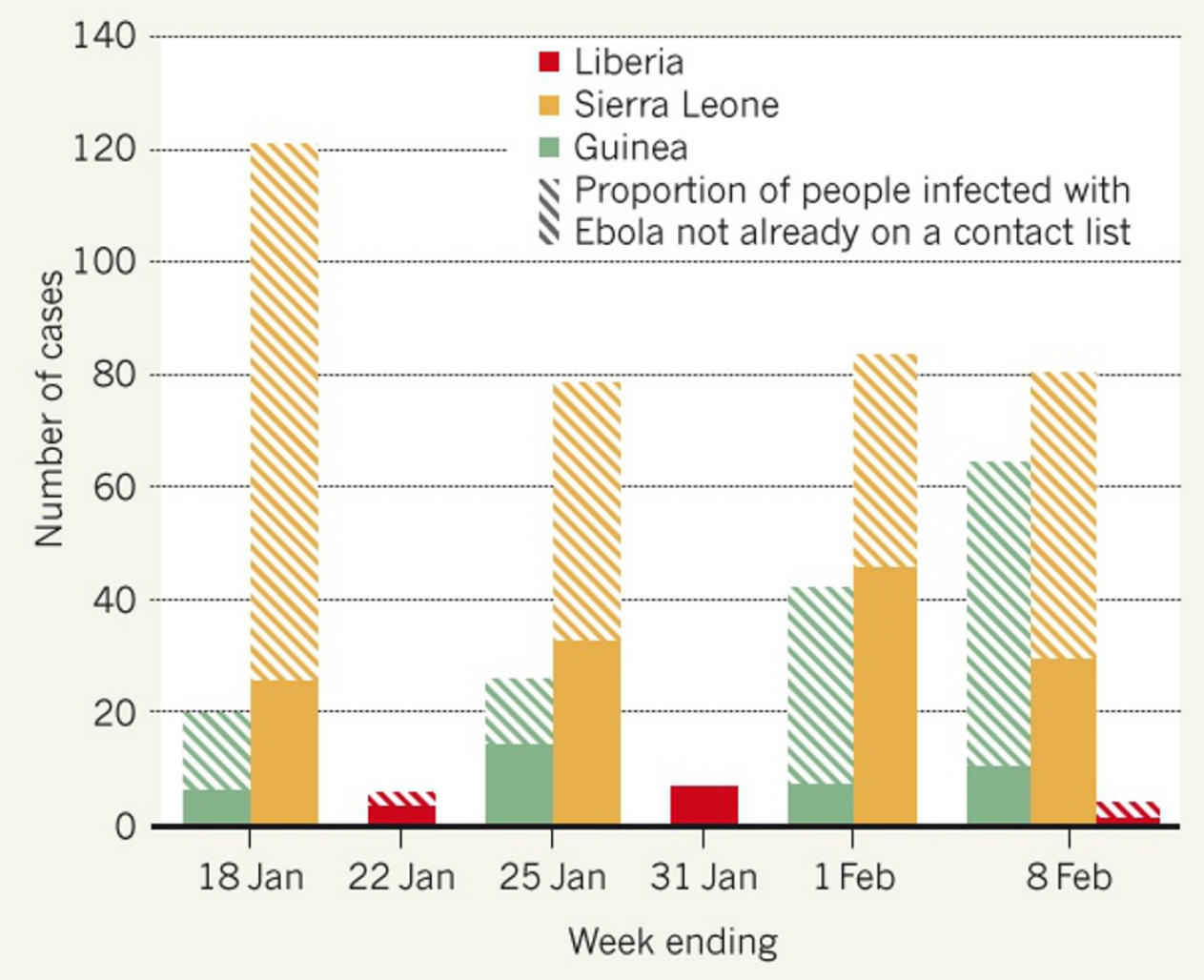

Source: $W H O$

\section{Shifting epidemiology}

The incidence, distribution and other features of Ebola outbreaks in West Africa are changing, posing new challenges. A few months ago, massive outbreaks called for large-scale humanitarian efforts, including the construction of many large Ebola treatment centres. Now, most outbreaks tend to be smaller, and in a more confined geographical area (see 'Ebola hotspots'). The current situation calls for a more varied and flexible response strategy. In places where the number of new cases is low, aggressive contact tracing becomes the priority, whereas in those where the number of cases is high, more resources still need to be allocated to treatment and ensuring safe burials.

Response efforts now need to target funds, supplies and staff to the particular epidemiological characteristics of each outbreak. This may prove challenging, because national and international health bureaucracies are not known for being nimble. "What will get us finished is what we call case finding and contact tracing," said Bruce Aylward, who leads World Health Organization's response on Ebola, at a press conference at the United Nations in New York on 20 February. "The big danger is that the international community doesn't make that shift in thinking and understanding." 


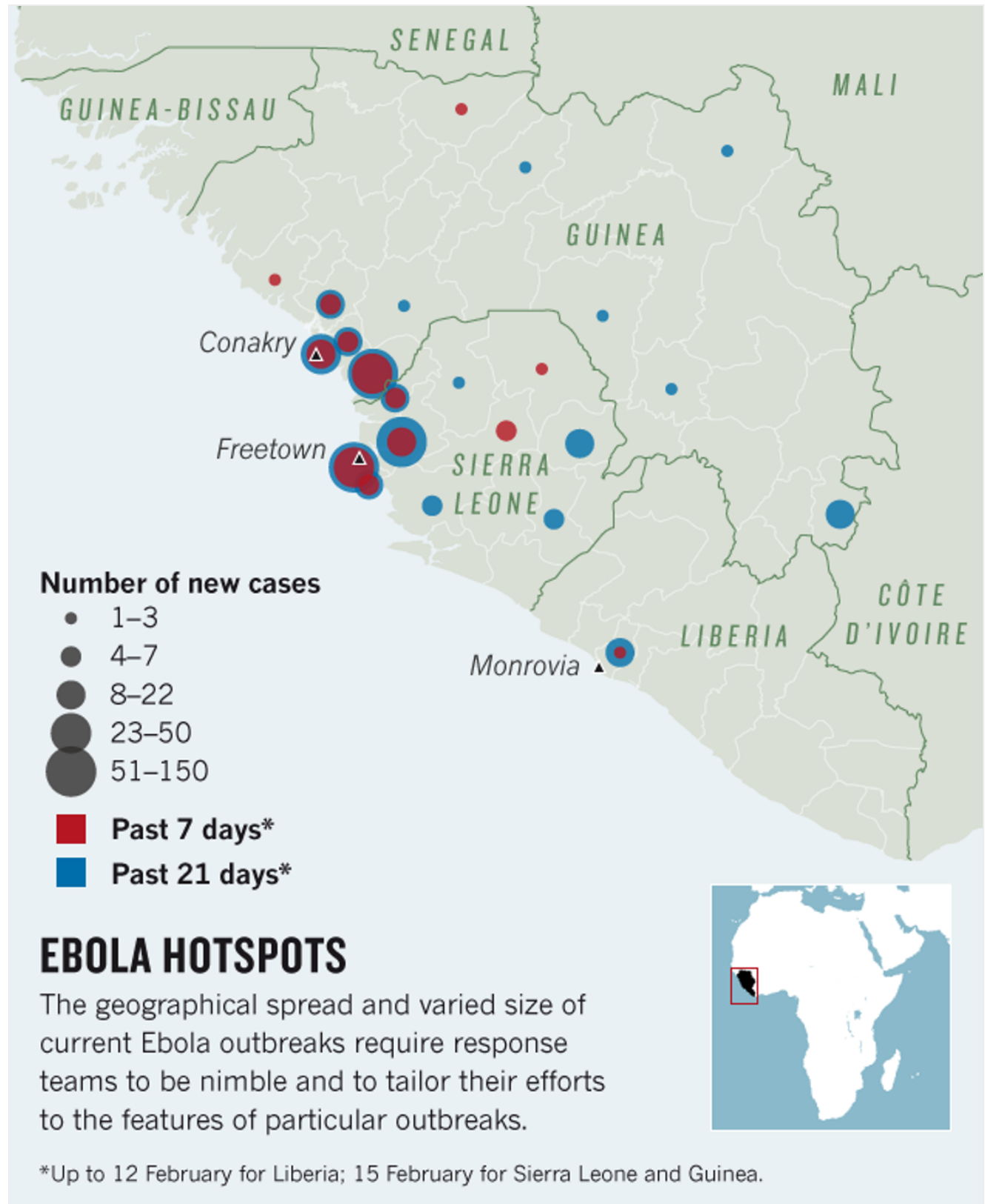

Source: $W H O$

\section{Dangerous burials}

Traditional burials of those who have died from Ebola are a major source of spread because they bring many of those attending into direct contact with infected corpses (see 'Dangerous funerals'). The World Health Organization (WHO) continues to report dozens of unsafe burials every week, well short of its target of zero. On 12 February, the International Red Cross and Red Crescent Movement also expressed concern over the beatings of two of its volunteers while organizing a safe burial in Forécariah, in western Guinea. It is part of a continuing pattern of verbal and physical assaults, in Guinea in particular, on Ebola workers by scared locals. 


\section{DANGEROUS FUNERALS}

The number of traditional burials involving direct contact with the corpse, which can spread Ebola, is well above the WHO target of zero in Guinea and Sierra Leone.

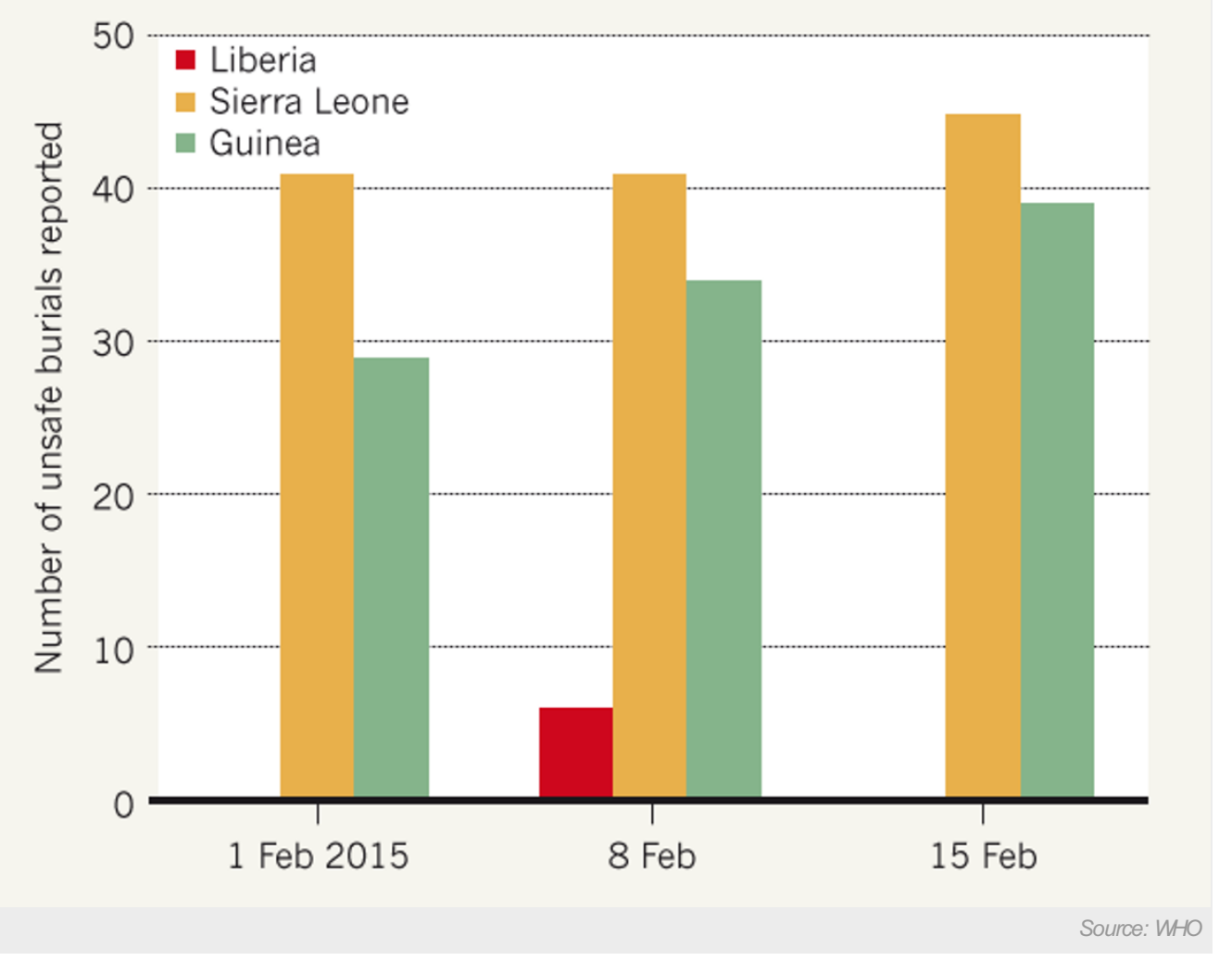

\section{Money and staff}

The United Nations estimates that its Ebola response will need US $\$ 1.5$ billion for the first six months of this year, but it has only $\$ 600$ million in hand, leaving a $\$ 900$ million shortfall, just at the time it wants to scale-up efforts. "One of the biggest risks we face right now is that new financing for this response is falling faster than the new cases," said Aylward. The UN also estimates that more than 2,171 international and 65,603 local staff will be needed for Ebola efforts over the same period.

\section{Rain}

The biggest obstacle to achieving zero Ebola cases anytime soon might be the weather. The rainy season in West Africa will kick off around the end of April, peak during the summer, and run until roughly the end of October. Once in full swing, it will reduce dirt tracks, which much of the more remote areas in West Africa rely on for transport, to thick mud. This will badly hamper — if not derail — almost every aspect of the outbreak response. "The virus has told us loud and clear this week: 'all I have to do is to survive out the next few weeks or months until the rains hit and then you are going to have a very very difficult situation'," said Aylward.

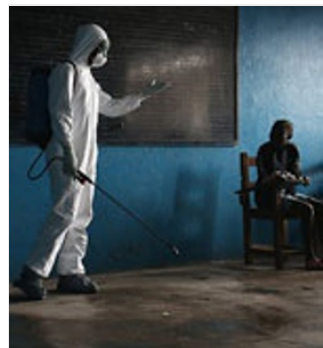

Nature special: Ebola outbreak in West Africa 


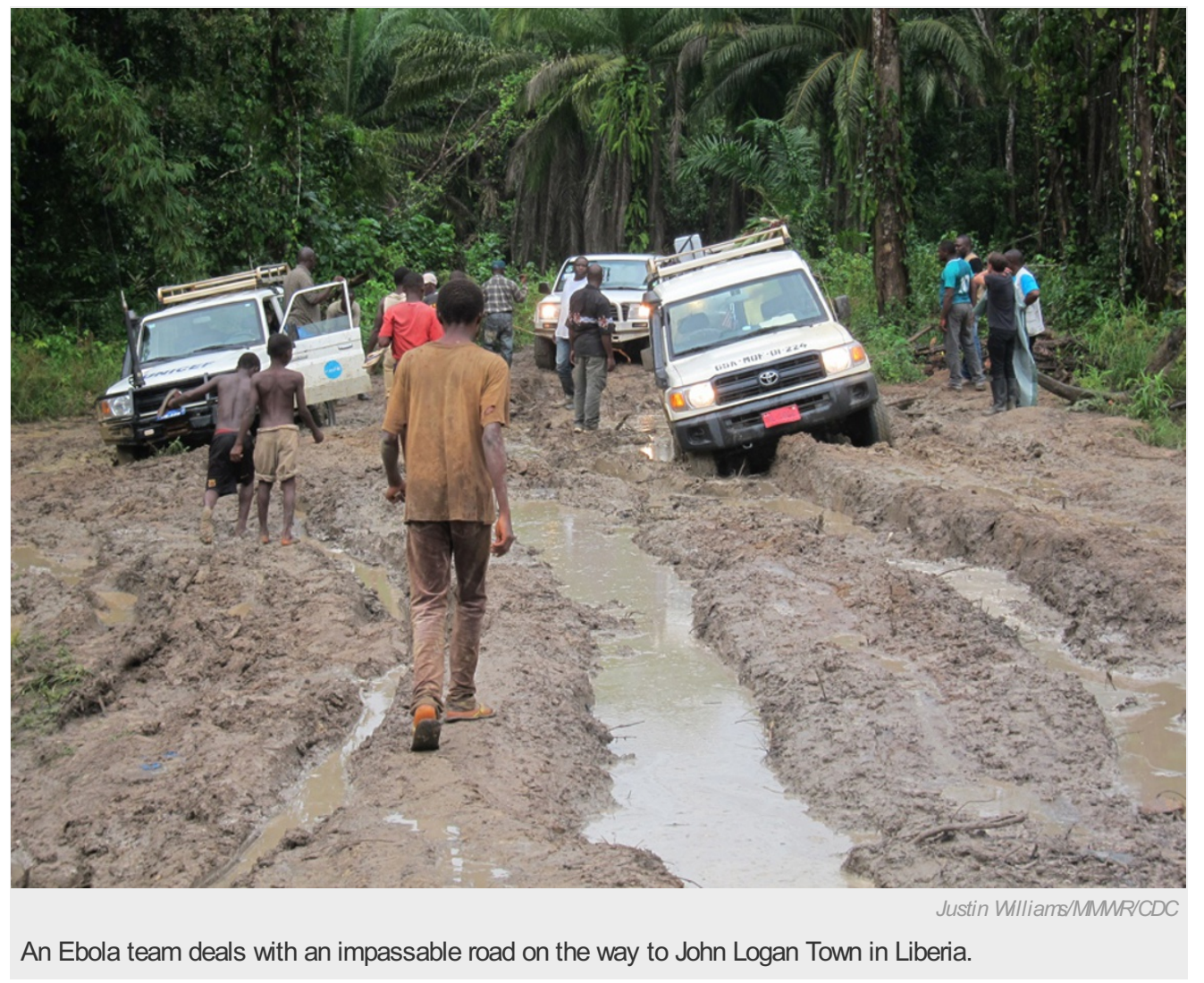

Nature | doi:10.1038/nature.2015.16964

\section{Corrections}

Corrected:An earlier version of the graphic 'Ebola hotspots' was based on out-of-date data. The map has now been updated.

Corrected:An earlier version of the graphic 'Hidden disease spread' referred to the low proportion of new Ebola cases in people who are not on the 'contact lists' of previously infected patients. It should have said high proportion. The graphic has now been corrected. 